\title{
Detection of Focal Cortical Dysplasia Lesions in MRI using Textural Features
}

\author{
Christian Loyek $^{1}$, Friedrich G. Woermann ${ }^{2}$, Tim W. Nattkemper ${ }^{1}$ \\ ${ }^{1}$ Applied Neuroinformatics, Faculty of Technology, Bielefeld University, Bielefeld \\ ${ }^{2}$ Bethel Epilepsy Center, Mara Hospital, Department MRI, Bielefeld \\ cloyek@techfak.uni-bielefeld.de
}

\begin{abstract}
Focal cortical dysplasia (FCD) is a frequent cause of medically refractory partial epilepsy. The visual identification of FCD lesions on magnetic resonance images (MRI) is a challenging task in standard radiological analysis. Quantitative image analysis which tries to assist in the diagnosis of FCD lesions is an active field of research. In this work we investigate the potential of different texture features, in order to explore to what extent they are suitable for detecting lesional tissue. As a result we can show first promising results based on segmentation and texture classification.
\end{abstract}

\section{Introduction}

Focal cortical dysplasia (FCD) [1], a disorganisation of cortical development, is an important cause of medically intractable partial epilepsy. In medically resistant epilepsy patients, only surgical removal of the dysplastic lesions leads to significant reduction or cessation of seizures. High-resolution magnetic resonance imaging (MRI) has improved detection of FCD in the past years and has allowed more patients to undergo resective surgery. However, visual analysis and identification of FCD is a challenging task and strongly depends on the experience and expertise of the observer.

In a large number of cases, FCD lesions can not clearly be distinguished from healthy cortex and often remain unrecognised in standard radiological analysis [2]. Even for experienced radiologists it is difficult to descry the subtle characteristics with which FCD lesions are displayed in MR images. This motivates the question whether a computerized quantitative analysis will be capable to reliably detect these lesions.

In a few previously published approaches it was shown that an observer independent, quantitative analysis of MRI data has potential to assist in the diagnostics of FCD [3, 4]. Even though the proposed methods increase the sensitivity for FCD, the results are often associated with an increased number of false positive (FP) results [5]. This is due to the fact that the proposed methods are based on visual MRI characteristics [6] which can not clearly be linked to FCD lesions.

In this work, we investigate the potential of machine learning based classification of texture features for the purpose of detecting local structures in MR 
Table 1. Features considered in this study can be separated into three groups

\begin{tabular}{lll}
\hline Statistical & Grey level co-occurrence & Grey level run-length \\
\hline$x_{1}^{\mathrm{S}}=$ mean & $x_{1}^{\mathrm{CO}}=$ contrast & $x_{1}^{\mathrm{RL}}=$ short run emphasis \\
$x_{2}^{\mathrm{S}}=$ median & $x_{2}^{\mathrm{CO}}=$ homogeneity & $x_{2}^{\mathrm{RL}}=$ long run emphasis \\
$x_{3}^{\mathrm{S}}=$ variance & $x_{3}^{\mathrm{CO}}=$ inverse difference & $x_{3}^{\mathrm{RL}}=$ grey level distribution \\
$x_{4}^{\mathrm{S}}=$ skewness & $x_{4}^{\mathrm{CO}}=$ energy & $x_{4}^{\mathrm{RL}}=$ run-length distribution \\
$x_{5}^{\mathrm{S}}=$ kurtosis & $x_{5}^{\mathrm{CO}}=$ entropy & $x_{5}^{\mathrm{RL}}=$ run percentage \\
$x_{6}^{\mathrm{S}}=$ energy & & \\
$x_{7}^{\mathrm{S}}=$ entropy & & \\
\hline
\end{tabular}

images. We demonstrate that our features are able to recover lesional tissue within single cases with a low number of false positives, even for cases which are not easy to identify for the expert eye.

\section{Materials and Methods}

MRI scanning was performed on a 1.5 Tesla scanner (Siemens Magnetom Symphony, Erlangen, Germany) equipped with a standard head coil. The analysis comprises T1-weighted 3D-sequences (MPRAGE, TR $=11.1 \mathrm{msec}$, TE $=4.3$ msec, slice thickness $1.5 \mathrm{~mm}$, FOV $201 \mathrm{~mm} \times 230 \mathrm{~mm}$, matrix $256 \times 256$, voxel size $0.9 \mathrm{~mm} \times 1.5 \mathrm{~mm} \times 0.9 \mathrm{~mm}$ ) which were provided by the Bethel Epilepsy Center in Bielefeld, NRW. All images include a histologically confirmed FCD lesion. Each lesion was manually labeled by an experienced neuroradiologist.

\section{$2.1 \quad$ Features}

One possibility to describe texture is to calculate statistical moments based on the grey level histogram of a region or a window. In literature, statistical features are often called first-order texture features. Seven statistical measures have been extracted according to [7] (table 1, first column).

To account for second order texture properties we additionally apply one of the most well-known methods of texture analysis: grey-level co-occurrence matrices (COM) proposed by Haralick et al. [8]. A COM counts the relative occurrences of voxel pairs in an image window that have certain intensity values and that are seperated by a voxel distance $d^{\mathrm{COM}}$ in a relative direction $\alpha^{\mathrm{COM}}$. Based on the cost statistics different features can be computed as described in [8]. Five of the most common COM features are selected (table 1, second column).

We further extend the set of features by using a run-length-based technique as proposed by Galloway [9]. It calculates characteristic textural measures from grey-level run lengths in different image directions $\alpha^{\mathrm{RLM}}$. The third column in table 1 shows the five scalar features derived from the grey-level run length matrices (RLM). 


\subsection{Experimental Setup}

For each analysed image an inner-case-classification-setup is performed. Innercase classification aims to determine to what extend the applied texture features are suitable to detect FCD lesions within a single image. The setup has three steps:

- Image Preprocessing: In order to exclusively consider voxels of the cortex, the images are previously segmented into grey matter (cortex), white matter and cerebrospinal fluid using a standard algorithm described in [10] implemented in the statistical parametric mapping software (SPM2). For the calculation of the co-occurrence and the run-length features, the number of image grey values were reduced to 32 values.

- Feature Extraction: Each cortex voxel $v \in \Omega$ with position $(l, m, n)$ is associated with a feature vector $\mathbf{x}_{l m n}$ which combines all scalar texture measures above-mentioned and can be formulated as

$$
\mathbf{x}_{l m n}=\left(x_{1}^{\mathrm{S}}, \ldots, x_{7}^{\mathrm{S}}, \bar{x}_{1}^{\mathrm{CO}}, \ldots, \bar{x}_{5}^{\mathrm{CO}}, \bar{x}_{1}^{\mathrm{RL}}, \ldots, \bar{x}_{5}^{\mathrm{RL}}\right)^{\mathrm{T}}
$$

All values of $\mathbf{x}_{l m n}$ are calculated within a $7 \times 5 \times 7$-window with $v(l, m, n)$ as the center voxel. In the case of the COM, we selected the distance $d^{\mathrm{COM}}=1$ and directions $\alpha^{\mathrm{COM}}=0^{\circ}, 45^{\circ}, 90^{\circ}, 135^{\circ}$ within coronal slices. This results in four COMs, each representing one special direction. Thus, for each COM a set of five derived features is obtained (table 1, second column). In order to make the features invariant to the angle $\alpha$, each feature value $x_{1}^{\mathrm{CO}}, \ldots, x_{5}^{\mathrm{CO}}$ of the four COMs is averaged and finally described by $\bar{x}_{1}^{\mathrm{CO}}, \ldots, \bar{x}_{5}^{\mathrm{CO}}$. The values $\bar{x}_{1}^{\mathrm{RL}}, \ldots, \bar{x}_{5}^{\mathrm{RL}}$ of $\mathbf{x}_{l m n}$ are obtained in the same way using $\alpha^{\mathrm{RLM}}=$ $0^{\circ}, 45^{\circ}, 90^{\circ}, 135^{\circ}$ for the run-length directions.

- Training 83 Classification: Voxels have to be classified depending on whether they represent the class of normal cortex (label $y=-1$ ) or the class of lesional cortex (label $y=1$ ). For this purpose, we randomly extracted a training dataset $\left.\Gamma=\Gamma_{\mathrm{N}} \cup \Gamma_{\mathrm{L}}=\left\{\mathbf{x}_{i}, y_{i}\right)\right\}, i=1, \ldots, k$ with $\Gamma_{\mathrm{L}}$ being a subset of lesional tissue signals and $\Gamma_{\mathrm{N}}$ being a subset of normal tissue signals. A support vector machine (SVM) with a gaussian kernel was trained using $\Gamma$. Finally, all cortex voxels $v \in \Omega \backslash \Gamma$ are classified based on the trained SVM.

\section{Results}

Our method is applied to five different cases, i.e. subjects. In each case the FCD lesion was marked by an expert (author F.G.W.). The accuracy is assessed by computing sensitivity (SE) and specificity (SP) as shown in table 2. Results for one particular case are illustrated in figure 1. Figure 1(a) shows a coronal slice of an original T1-weighted image including the expert label. Our method generates three-dimensional classification maps which visualise the class of the voxels assigned by the SVM (figure 1(b)), white regions indicate lesional characteristics whereas grey voxels represent tissue classified as normal. Figure 1(c) shows an overlay of the original image and regions classified as lesional tissue. 
Table 2. The sensitivity (SE) and specificity (SP) values of the classification of five different cases

\begin{tabular}{llllll}
\hline & subject 1 & subject 2 & subject 3 & subject 4 & subject 5 \\
\hline SE & 0,946 & 0,904 & 0,846 & 0,960 & 0,982 \\
SP & 0,865 & 0,892 & 0,734 & 0,880 & 0,922 \\
\hline
\end{tabular}

\section{Discussion}

We propose a quantitative approach using local texture features for the innercase detection of focal cortical dysplasia lesions in MRI. The used features are powerful enough to allow automatic discrimination of normal cortex and lesional cortex tissue. In this work the proposed classification setup is only applied on an inner-case study to verify whether the features are suitable to find a lesion, however within the same image.

Using texture features we successfully detect lesional tissue in each of the applied cases which is confirmed by the increased SE values. Figure 1(b) and 1(c) exemplarily point out that the main part of the lesion is correctly classified. Furthermore, figure 1(b) demonstrates that only a small fraction of voxels is classified as false positives (see table 2 for sensitivity and specificity values for the remaining cases).
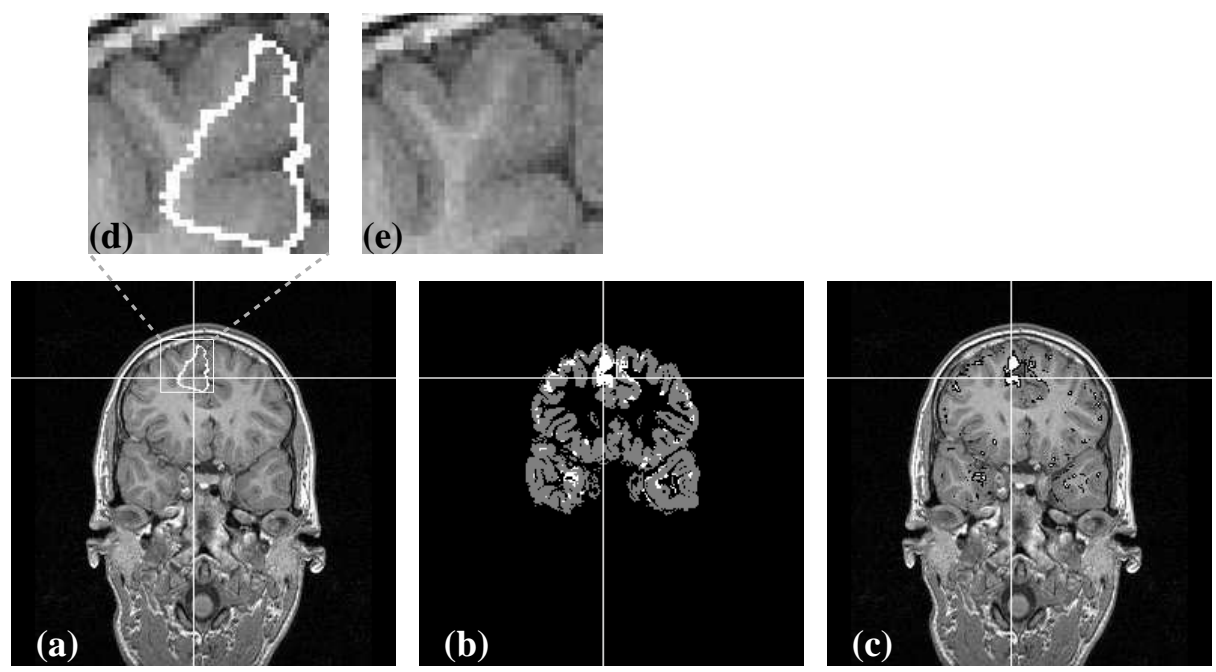

Fig. 1. Images describing results of subject 2. (a) Slice of the original T1-weighted MRI including a marking hand-labeled by an expert. (b) Classification map. (c) Overlay of the original image and the regions classified as lesional tissue. (d) and (e) show a magnification of the lesion 
The classification setup is applied on previously segmented cortex tissue which is of particular importance for the classification accuracy. In the beginning of the study, the whole image was used for training the SVM, but this led to significantly inferior classification results. A major fraction of image information which was learned by the SVM is not important for this classification problem.

Different neighborhood sizes for the feature extraction are tested. A window size of $7 \times 5 \times 7$ leads to the best results. In order to overcome the problem of voxel anisotropy, only five slices in $\mathrm{y}$-direction are considered.

The quantisation of the images in order to compute the COM and RLM features leads to a major performance increasement than using the original number of grey values. The conclusion that follows is that two much details, i.e. number of grey values, suppress the general differences between the types of tissue.

We perform the classification based on single feature approaches, i.e. statistical features, COM features and RLM features (see columns in table 1). The results point out that using single feature methods leads to an inferior classification accuracy than merging the feature sets. As a conclusion, FCD lesions contain both first- and second-order properties which enable their discrimination from normal cortex tissue. The results of this work can be considered as a necessary basic condition for realising a real CAD (computer aided diagnosis) setup which has to be analysed regarding generalisation in future studies.

Acknowledgement. The work is funded by the Bielefeld University.

\section{References}

1. Taylor D, Falconer M, Bruton C, et al. Focal dysplasias of the cerebral cortex in epilepsy. J Neurol Neurosurg Psychiatry. 1971;34:369-87.

2. Tassi L, Colombo N, Garbelli R, et al. Focal cortical dysplasia: Neuropathological subtypes, EEG, neuroimaging and surgical outcome. Brain. 2002;125:1719-32.

3. Huppertz H, Grimm C, Fauser S, et al. Enhanced visualization of blurred graywhite matter junctions in focal cortical dysplasia by voxel-based 3D MRI analysis. Epilepsy Res. 2005;67:35-50.

4. Colliot $\mathrm{O}$, Antel $\mathrm{S}$, Naessens $\mathrm{V}$, et al. In vivo profiling of focal cortical dysplasia on high-resolution MRI with computational models. Epilepsia. 2006;47(1):134-42.

5. Woermann F, Brandt C, Schaumann-von Stosch R. Neuroradiologische Diagnostik in der Epileptologie. Akt Neurol. 2004;31:60-72.

6. Barkovich A. Pediatric Neuroimaging. Williams + Wilkins, Philadelphia: Lippincott; 2000.

7. Pratt W. Digital Image Processing. New York: John Wiley \& Sons; 1991.

8. Haralick R, Shanmugam K, Dinstein I, et al. Textural features for image classification. IEEE Trans System Man Cybern. 1973;3(6):610-21.

9. Galloway M. Texture analysis using gray level run lengths. Comp Graph Image Process. 1975;4:172-9.

10. Ashburner J, Friston K. Multimodal image coregistration and partitioning: A unified framework. Neuroimage. 1997;6:209-17. 